\title{
Advice to use infant formula and free samples are common in both urban and rural areas in China: a cross-sectional survey
}

Jia Li ${ }^{1} \odot$, Tuan T Nguyen ${ }^{2} \odot$, Yifan Duan ${ }^{3} \odot$, Roger Mathisen ${ }^{2} \odot$ and Zhenyu Yang ${ }^{3, *}$ () 'School of Business, Nanjing University of Information Science \& Technology, Nanjing, People's Republic of China: ${ }^{2}$ Alive \& Thrive Southeast Asia, FHI 360, Hanoi, Vietnam: ${ }^{3}$ National Institute for Nutrition and Health, Chinese Center for Disease Control and Prevention, No.27 Nanwei Road, Xicheng District, Beijing 100050, People's Republic of China

Submitted 1 September 2020: Final revision received 24 November 2020: Accepted 21 December 2020: First published online 8 January 2021

\begin{abstract}
Objective: To examine the association between the place of residence and receiving free samples and advice to feed the baby with infant formula.

Design: A cross-sectional study.

Setting: The current study covered twelve counties/districts in China.

Participants: 5112 mothers with infants aged 0-5.9 months.

Results: About $16 \%$ of the mothers received free samples of infant formula. During pregnancy, this likelihood was higher among mothers in small and medium cities (OR: 1.96; $95 \%$ CI 1.14, 3.38) and non-poor rural counties (OR: 4.65; $95 \%$ CI 1.65, 13.14) compared with mothers in big cities. During the hospital stay, it was lower in big cities. After discharge, it was lower in poor rural counties (OR: 0.14; $95 \%$ CI $0 \cdot 05,0 \cdot 41$ ). About $26 \%$ of the mothers were advised to feed their infants with infant formula. The likelihood of receiving advice to feed the baby with infant formula from hospitals was lower in non-poor (OR: 0.37; $95 \%$ CI 0.21, 0.66) and poor rural counties (OR: $0 \cdot 35 ; 95 \%$ CI $0 \cdot 13,0.91$ ) than in big cities. Mothers in non-poor rural counties were less likely to receive advice from traditional mass media (OR: $0 \cdot 17$; $95 \%$ CI $0 \cdot 06,0 \cdot 48$ ), while mothers in small and medium cities were more likely to receive advice from modern mass media (OR: 1·84; $95 \%$ CI 1.20, 2.80) compared with mothers in big cities.

Conclusions: The promotion strategy of infant formula varies from different places of residence in China. The study suggests the need to strengthen enforcement of relevant regulations, especially within health facilities and through modern mass media.
\end{abstract}

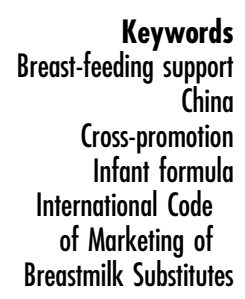

Aggressive promotion of breastmilk substitutes (BMS) is one of the key barriers to successful breast-feeding and thus poses dangers to infant health ${ }^{(1,2)}$. Previous studies showed that inappropriate marketing of BMS affects breast-feeding behaviours of women as well as medical practice of healthcare workers related to supporting breast-feeding $^{(3-5)}$. For example, misconceptions related to feeding and intention to feed infant formula at birth were associated with increased feeding of infant formula in the first $3 \mathrm{~d}$ of life, which is associated with increased feeding of infant formula and premature cessation of breastfeeding ${ }^{(6)}$. Studies in China also revealed that mothers who received advice to feed the baby with BMS had a lower prevalence of exclusive breast-feeding and continued breast-feeding ${ }^{(7,8)}$. Promotion of infant formula hinders progress towards achieving the Global Nutrition Targets and Sustainable Development Goals endorsed by most governments $^{(9)}$.

Infant formula sales are high and increasing in East Asia, including in China ${ }^{(3,10,11)}$. Indeed, China is the largest infant formula market in the world ${ }^{(3)}$. The sale of baby food in China, around $90 \%$ of which was formula milk, doubled in just 5 years from 2010 to 2014, and then one and a half fold from 2014 to $2018^{(12,13)}$. BMS manufacturers and distributors employ various tactics to promote their products. In addition to direct promotion to mothers and their families via television, social media and home visits, manufacturers and distributors promote BMS indirectly via incentives, free samples and activities within the health service setting during pregnancy, hospital stay and after 
discharge $\mathrm{e}^{(1,4,14-17)}$. Distribution of free samples of infant formula or promotional materials to pregnant women typically happens during consultations and events organised by BMS manufacturers and distributors, where they familiarise women with the products and aim to increase the preference for certain types of infant formula to use in the future ${ }^{(18,19)}$. The promotion of infant formula during the hospital stay and after discharge through health workers and BMS representatives is also common in China ${ }^{(20,21)}$. Additionally, cross-promotion (e.g., similar labelling, ambiguous messages) across BMS product categories (e.g., milk formula for pregnant women, preterm babies, infants and toddlers) is a common practice of BMS manufacturers and distributors to circumvent the national Code legislation, creating confusion for families ${ }^{(2)}$.

Given the negative impact of BMS promotion and aggressive marketing tactics, regulating the inappropriate practices of BMS companies has been identified as a critical intervention to protect and build supportive environments for breast-feeding ${ }^{(22)}$. The World Health Assembly adopted the International Code of Marketing of Breastmilk Substitutes (hereafter the Code) in 1981 to prohibit the advertising and promotion of BMS ${ }^{(23)}$. Even though 136 out of 194 countries have adopted some forms of national legal measures adhering to the Code by 2020, robust measures are in place in only a few countries to eliminate inappropriate promotion of $\mathrm{BMS}^{(24)}$. According to this report, only some provisions of the Code are included in the current regulations in China ${ }^{(24)}$. The main policy, Administrative Measures for the Marketing of Breastmilk Substitutes (hereafter the Measure) issued in 1995(25), was abolished in 2017, leaving the marketing of BMS in China weakly regulated (online supplementary Appendix A).

Furthermore, the China Food and Drug Administration introduced a new regulation in 2016 to establish standards for infant formula ${ }^{(26,27)}$, creating opportunities for certain brands to market themselves as high quality to help expand their market into medium and small cities and rural areas where substandard products were commonly used ${ }^{(28)}$. As the Chinese economy develops, rising income makes infant formula more affordable for mothers in lower socioeconomic groups (e.g., rural or urban poor). Introduction of the universal two-child policy in 2015 is projected to result in a significant increase in birth rate, especially in small cities and rural areas of China ${ }^{(29)}$. BMS companies might have captured those policy gaps and sociodemographic dynamic changes to expand their market in China. Previous studies in China have focused on the promotion of infant formula in big cities ${ }^{(7,20)}$. Thus, information about the promotion of infant formula in lessurbanised areas in China is limited.

To address this gap in the literature, we analysed data from a large-scale population survey to examine the association between the place of residence and receiving free samples and advice to feed the baby with infant formula among mothers with infants aged 0-5.9 months. We hypothesised that the promotion of BMS varied across places of residence.

\section{Methods}

\section{Study design and data collection}

In the current study, we used secondary data from 10408 mothers with infants aged 0-11 months old who participated in a cross-sectional survey on determinants of breast-feeding practices in China ${ }^{(8)}$. Infants were approximately equally distributed across each month group. A more detailed description of the study has been described elsewhere $^{(8)}$. To summarise, survey samples were selected via a multi-stage stratified cluster sampling approach. All districts/counties were categorised into four strata, namely big cities, small and medium cities, non-poor rural counties and poor rural counties. Among twelve districts/counties selected in the first stage, four were from big cities, four were from small and medium cities, two were from nonpoor rural counties and two were from poor rural counties. In the second stage, four clusters were randomly selected via probability proportional to size sampling method. In each selected cluster, the data collection team visited the corresponding immunisation clinic and invited mothers who brought their 0-11-month-old children to the clinic for immunisation to participate in the study. In total, the survey included face-to-face interviews of 10408 mothers with infants aged 0-11 months old.

Data were collected between September 2017 and January 2018 in collaboration with research teams at the provincial level Center for Disease Control and Prevention. A structured questionnaire was programmed into smartphones or tablets and then used for data collection (online supplementary Appendix B). Written consent from all participants was obtained.

\section{Study variables}

\section{Outcome variables}

Promotion of infant formula in the current study was defined as having received free samples of infant formula or advice to feed the baby with infant formula, which was assessed using two retrospective questions. The first question was 'when did the mothers receive free samples of infant formula?' We examined three time points: (1) during pregnancy, (2) during hospital stay and (3) after discharge as well as an overall measure of receiving free samples of infant formula (No, when the mother did not receive any free samples of infant formula in any of the three time points; and Yes, for any alternative scenarios). The second question was whether the mothers received advice to feed the baby with infant formula, and if yes, from where. We examined four circumstances: (1) from hospitals where the mother gave birth, (2) from traditional mass media 
(e.g., TV, radio, magazine or book), (3) from modern mass media (e.g., websites, online shopping malls, websites and platform from hospitals or doctors, and social media such as Weibo and WeChat) and (4) from family members, relatives or friends.

\section{Exposure variables}

The place of residence was the main exposure in the current study. The whole of China can be divided into twentythree provinces, five autonomous regions, four centrally administered municipalities and two special administrative regions, which are then subdivided into prefectures, counties/districts and townships ${ }^{(30,31)}$. We sampled districts/counties from big cities, small and medium cities, non-poor rural counties and poor rural counties. Big cities refer to central districts of municipalities directly under the Central Government, cities under separate state planning or provincial cities with a population over a million. Small and medium cities refer to all districts/counties except the central districts of big cities and county-level cities. Poor rural counties refer to counties that are key targets in the national poverty alleviation and development programme ${ }^{(32)}$. Non-poor rural counties refer to all remaining counties.

\section{Covariate variables}

Maternal and paternal characteristics include level of education (primary or below, junior high school, high school or college and university or higher) and occupation (e.g., unemployed, agriculture related, industry related and white-collar or professionals). Maternal age ( $\leq 25,26-35$ and $\geq 36$ years) and ethnicity (Han and others) were also collected. Child and perinatal characteristics consist of age in months, gender, first birth, having at least one antenatal visit, place of birth (maternity facilities at national or provincial levels, at municipal level, at county level and others), caesarean birth, length of stay in health facility after birth if extended (vaginal births: $\geq 4 \mathrm{~d}$; caesarean births: $\geq 7$ d) and special care service for the mothers such as hiring a nanny or staying in a postpartum care centre during the first month afterbirth ${ }^{(33)}$. For the first set of outcome variables on receiving free samples of infant formula, we control for all of the above-mentioned covariates. For the second set of outcome variables about receiving advice to feed the baby with infant formula, we also consider additional covariates including reasons for being advised on feeding infant formula (perceived breast-feeding difficulties in hospital and after discharge) and hospital lack of referral for breast-feeding supporting organisations at discharge.

\section{Statistical analysis}

From 10408 mothers interviewed, we selected 5261 mothers with infants aged $0-5.9$ months. Then, we further excluded 149 records ( $<3 \%$ of 5261 records) due to missing values in outcome variables and covariates to come up with the final sample for statistical analysis of 5112 . Sensitivity analysis with the complete sample displayed very similar findings with those reported in the current study. The selection of mothers with infants aged 0-5.9 months was to minimise recall bias of the exposure to infant formula free sample and advice.

All the data analysis was conducted using survey command in Stata 15.0 (Stata Inc.) to consider survey design effect. First, we used descriptive analyses to report the prevalence of exposure and covariate variables, and stratified outcome variables by the place of residence. Then, we used multivariate logistic regression models to examine factors associated with receiving free samples or receiving advice to feed the baby with infant formula. We used big cities, mothers with a university education or higher, and mothers who worked as white-collar workers or professionals as the reference groups in the multivariate logistic regression models.

\section{Results}

\section{General characteristics}

Among 5112 mothers with infants 0-5 months old (mean age of 2.5 months), $86.1 \%$ belonged to the Han ethnicity, $63.3 \%$ were between 26 and 35 years old, $21.4 \%$ had at least a college degree and $44.7 \%$ participated in business, professional or industry-related occupations (Table 1). Mothers in rural areas tended to be younger, had less education and were less likely to work as white-collar workers or professionals (Table 1). Fathers of infants had similar educational and occupational patterns as mothers (Table 1).

About half of the sample were boys and $46.8 \%$ were the first child. Almost all mothers had antenatal care, delivered at a health facility and spent some days after births at the health facility. The prevalence of caesarean births was $38.8 \%$ and $32.5 \%$ of the mothers had an extended stay in a maternity facility after delivery (vaginal births: $\geq 4 \mathrm{~d}$; caesarean births: $\geq 7 \mathrm{~d}$ ).

About a quarter of mothers felt they had difficulty with breast-feeding during the hospital stay or after discharge. Less than $10 \%$ of the mothers hired a nanny or stayed in a postpartum care centre during the first month after birth, and the majority of whom lived in big cities. Only one of every ten mothers was referred to a breast-feeding supporting centre at discharge.

\section{Receiving free samples of infant formula}

About $16 \%$ of mothers received free samples of infant formula: $6 \cdot 3 \%$ received the sample during pregnancy, $3.5 \%$ during their hospital stay and $6.3 \%$ after discharge (Fig. 1). Although there were some variations by the place of residence, the $95 \% \mathrm{CI}$ were overlapping in most 
Table 1 Characteristics of participants (in percentage)

\begin{tabular}{|c|c|c|c|c|c|}
\hline & $\begin{array}{l}\text { All sample } \\
(n 5112)\end{array}$ & $\begin{array}{l}\text { Big cities } \\
(n \text { 1797) }\end{array}$ & $\begin{array}{l}\text { Medium and small } \\
\text { cities }(n 1663)\end{array}$ & $\begin{array}{l}\text { Non-poor rural } \\
\text { areas }(n 833)\end{array}$ & $\begin{array}{c}\text { Rural poor } \\
\text { areas }(n 819)\end{array}$ \\
\hline \multicolumn{6}{|l|}{ Parental characteristics } \\
\hline \multicolumn{6}{|l|}{ Maternal ethnicity } \\
\hline Han & $86 \cdot 1$ & $84 \cdot 1$ & $80 \cdot 3$ & $99 \cdot 0$ & 88.9 \\
\hline \multicolumn{6}{|l|}{ Maternal age } \\
\hline$\leq 25$ years & $25 \cdot 2$ & $13 \cdot 0$ & 30.4 & $29 \cdot 1$ & $37 \cdot 7$ \\
\hline $26-35$ years & 63.3 & $69 \cdot 7$ & $60 \cdot 4$ & $65 \cdot 3$ & 53.4 \\
\hline$\geq 36$ years & 11.4 & $17 \cdot 3$ & $9 \cdot 3$ & $5 \cdot 6$ & 8.9 \\
\hline \multicolumn{6}{|l|}{ Maternal education levels } \\
\hline University or higher & $21 \cdot 4$ & $44 \cdot 1$ & $15 \cdot 2$ & $3 \cdot 1$ & $3 \cdot 1$ \\
\hline High school or college & $36 \cdot 5$ & $39 \cdot 0$ & $40 \cdot 2$ & $40 \cdot 9$ & $19 \cdot 3$ \\
\hline Junior high school & 34.9 & $15 \cdot 1$ & $34 \cdot 3$ & $54 \cdot 0$ & $60 \cdot 1$ \\
\hline Primary or below & $7 \cdot 1$ & 1.8 & $10 \cdot 3$ & 1.9 & $17 \cdot 6$ \\
\hline \multicolumn{6}{|l|}{ Maternal occupations } \\
\hline White-collar or professionals & $36 \cdot 0$ & $57 \cdot 1$ & 33.0 & 21.5 & $10 \cdot 4$ \\
\hline Industry related & 8.7 & $9 \cdot 6$ & $9 \cdot 8$ & 8.8 & $4 \cdot 2$ \\
\hline Agriculture related & $17 \cdot 3$ & $2 \cdot 8$ & $15 \cdot 0$ & $16 \cdot 6$ & $54 \cdot 3$ \\
\hline Unemployed related & 38.1 & 30.5 & $42 \cdot 2$ & 53.2 & 31.1 \\
\hline \multicolumn{6}{|l|}{ Paternal education levels } \\
\hline University or higher & $22 \cdot 1$ & $46 \cdot 4$ & 14.9 & $2 \cdot 8$ & $2 \cdot 9$ \\
\hline High school or college & $35 \cdot 3$ & $38 \cdot 1$ & $38 \cdot 1$ & $35 \cdot 8$ & $23 \cdot 2$ \\
\hline Junior high school & $35 \cdot 8$ & $13 \cdot 8$ & 37.8 & 57.4 & $58 \cdot 1$ \\
\hline Primary or below & $6 \cdot 8$ & 1.7 & $9 \cdot 3$ & 4.1 & $15 \cdot 8$ \\
\hline \multicolumn{6}{|l|}{ Paternal occupations } \\
\hline White-collar or professionals & $48 \cdot 2$ & $70 \cdot 0$ & $47 \cdot 1$ & $35 \cdot 3$ & $15 \cdot 9$ \\
\hline Industry related & 28.4 & 23.3 & 28.9 & $45 \cdot 6$ & $21 \cdot 0$ \\
\hline Agriculture related & 18.5 & $2 \cdot 9$ & $18 \cdot 2$ & $17 \cdot 4$ & $54 \cdot 6$ \\
\hline Unemployed related & 4.9 & $3 \cdot 8$ & $5 \cdot 8$ & $1 \cdot 7$ & 8.5 \\
\hline \multicolumn{6}{|l|}{ Child and perinatal characteristics } \\
\hline The first birth & $46 \cdot 8$ & $52 \cdot 9$ & $47 \cdot 8$ & 32.5 & $45 \cdot 9$ \\
\hline The child was male & $50 \cdot 2$ & $50 \cdot 4$ & 50.5 & $50 \cdot 1$ & $49 \cdot 2$ \\
\hline \multirow{2}{*}{\multicolumn{6}{|c|}{ Health facilities the mothers gave births }} \\
\hline & & & & & \\
\hline National and provincial levels & $18 \cdot 5$ & $44 \cdot 8$ & $4 \cdot 0$ & 0.4 & 8.7 \\
\hline Municipal level & $29 \cdot 3$ & 40.5 & $38 \cdot 2$ & $10 \cdot 2$ & $6 \cdot 1$ \\
\hline County level & $50 \cdot 3$ & $10 \cdot 1$ & $57 \cdot 5$ & 88.2 & $85 \cdot 2$ \\
\hline Others (mostly private) & 1.9 & $4 \cdot 7$ & 0.3 & $1 \cdot 2$ & 0.0 \\
\hline Caesarean delivery & $38 \cdot 8$ & 33.9 & 43.2 & $48 \cdot 0$ & 30.9 \\
\hline Long stay in health facility after births* & $32 \cdot 5$ & $27 \cdot 8$ & $29 \cdot 0$ & $15 \cdot 6$ & $67 \cdot 0$ \\
\hline Had breastfeeding difficulties in hospital & $26 \cdot 3$ & $36 \cdot 4$ & 21.9 & $20 \cdot 8$ & $18 \cdot 7$ \\
\hline Had breast-feeding difficulties after discharge & $26 \cdot 5$ & $39 \cdot 8$ & $20 \cdot 0$ & $20 \cdot 0$ & $17 \cdot 3$ \\
\hline $\begin{array}{l}\text { Hired a nanny or stayed in a postpartum care } \\
\text { centre during the first month afterbirth }\end{array}$ & $7 \cdot 6$ & $16 \cdot 6$ & 4.9 & $1 \cdot 1$ & $0 \cdot 1$ \\
\hline $\begin{array}{l}\text { Hospital where the woman gave birth did not } \\
\text { recommended breast-feeding supporting } \\
\text { centre at discharge }\end{array}$ & $92 \cdot 4$ & $88 \cdot 2$ & $96 \cdot 1$ & 98.7 & $87 \cdot 9$ \\
\hline
\end{tabular}

*Long stay in health facility after births: vaginal births: $\geq 4 \mathrm{~d}$; caesarean births: $\geq 7 \mathrm{~d}$.

pairwise comparisons, except for free infant formula sample after discharge: $1 \%$ of mothers in rural poor counties $v$. $4 \%$ in small and medium cities and $5 \%$ in non-poor rural counties (Fig. 1).

In multivariate logistic regression models, the likelihood of receiving free samples of infant formula during pregnancy was higher for mothers in medium and small cities (OR: 1.96; $95 \%$ CI 1.14, 3.38) and in non-poor rural counties (OR: 4.65; $95 \%$ CI $1.65,13.14$ ) than in big cities (Table 2). This result indicated that mothers in non-poor rural counties were more than four times more likely to receive free samples than those in large cities. Compared with mothers in big cities, mothers in other areas were more likely to receive free samples of infant formula during hospital stay; however, this difference was only significant in non-poor rural counties (OR:
2.06; $95 \%$ CI 1.04, 4.09). In contrast, mothers in big cities were more likely to receive free samples after discharge, especially when compared with mothers in poor rural counties (OR: 0.14; $95 \%$ CI 0.05-0.41) (Table 2). The likelihood of receiving free samples after discharge was higher in mothers working in agricultural sector (OR: 1.99; 95\% CI $1 \cdot 12,3 \cdot 54$ ) or unemployed (OR: $1.59 ; 95 \%$ CI 1.24, 2.05) than white-collar workers or professionals. The likelihood of mothers who received free samples of infant formula after discharge from private hospitals was higher than received by mothers who gave birth in maternity facilities at national and provincial levels (OR: 1.74; $95 \%$ CI 1.07, 2.84), but lower than received by mothers in maternity facilities at the county (OR: 0.41; $95 \%$ CI 0.26, 0.63) and municipal (OR: 0.51; $95 \%$ CI $0 \cdot 29,0 \cdot 89)$ levels. 


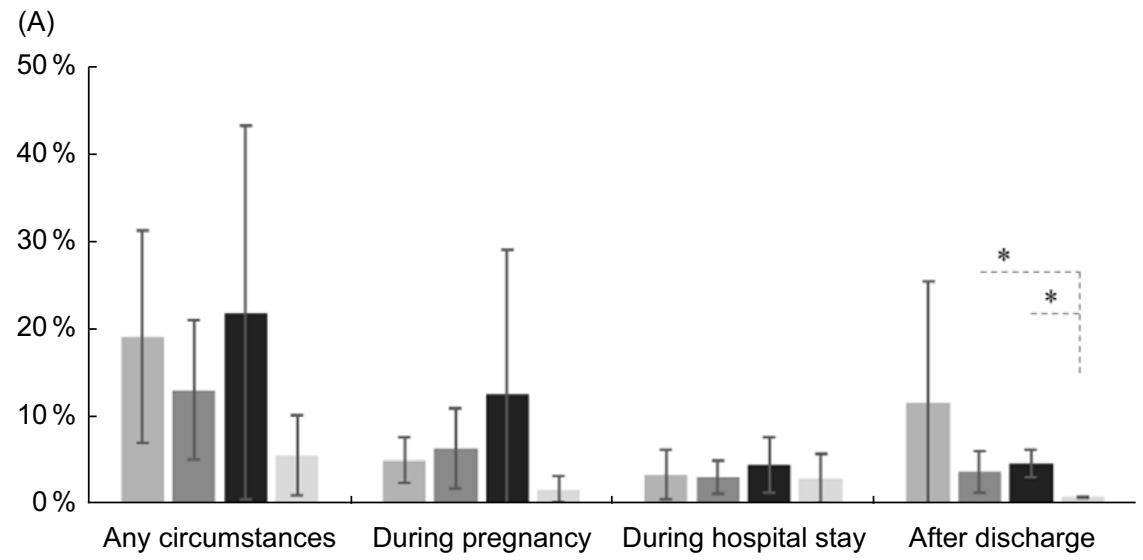

(B)

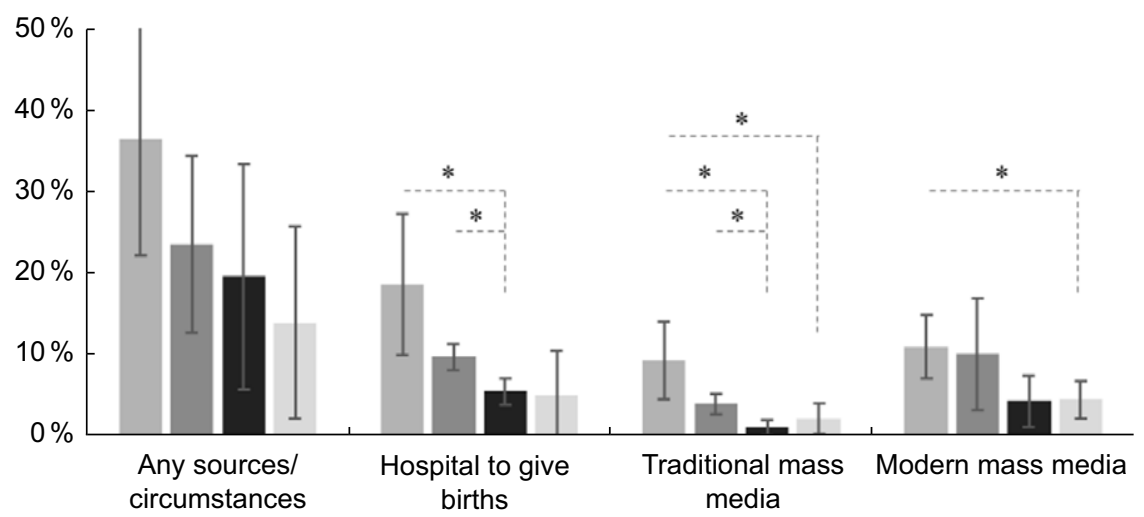

Fig. 1 Prevalence $(\%, 95 \% \mathrm{Cl})$ of receiving free samples of infant formula $(A)$ and advice $(B)$ among mothers with infants $<6$ months old in China by the place of residence * for non-overlapping $95 \% \mathrm{Cl}$. Traditional mass media: TV, radio, magazine or book; modern mass media: Websites, online shopping malls, websites and platform from hospitals or doctors, and social media such as Weibo and

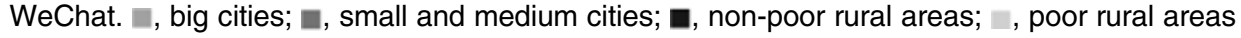

\section{Receiving advice to feed the baby with infant formula}

About $26 \%$ of mothers in this sample received advice to feed the baby with infant formula: $11.4 \%$ from hospitals, $5 \cdot 1 \%$ from traditional mass media like TV, radio, magazines and books, $8.6 \%$ from modern mass media such as the internet and social media and $3.1 \%$ from family members, relatives or friends (Fig. 1). More mothers in big cities received this advice from the hospitals and traditional mass media (e.g., TV, radio, magazine and books) than those living in other areas (Fig. 1). Receiving the advice from modern mass media such as internet and social media was higher in big cities than in rural poor counties (nonoverlapping 95\% CI) (Fig. 1).

Multivariate logistic regression models showed that the likelihood of receiving advice to feed the baby with infant formula from hospitals was lower in non-poor rural counties (OR: 0.37; $95 \%$ CI 0.21, 0.66) and poor rural counties (OR: $0.35 ; 95 \%$ CI $0.13,0.91$ ) than in big cities (Table 3 ). The likelihood of receiving advice to feed the baby with infant formula from mass media was lower in non-poor rural counties for traditional mass media (OR: 0.17 ; $95 \%$ CI $0.06,0.48$ ) but higher in small and medium cities for modern mass media (OR: 1.84; $95 \%$ CI 1.20, $2 \cdot 80)$ compared with mothers in big cities. The likelihood of receiving advice to feed the baby with infant formula was similar across most education levels, while it slightly differed across occupations of mothers and fathers (Table 3). Notably, mothers working in industry were more likely to receive advice to feed the baby with infant formula from modern mass media (OR: 1.53 ; $95 \%$ CI 1.23, 1.91) and family members, relatives or friends (OR: 1.66; $95 \%$ CI1.02, $2 \cdot 72$ ), and fathers working in industry (OR: 1.44; $95 \% \mathrm{CI}$ $1 \cdot 10,1 \cdot 89)$ for traditional mass media compared with those working as white-collar workers or professionals. The prevalence of receiving advice to feed the baby with infant formula was higher in mothers giving birth in maternity facilities at national and provincial levels than in other level facilities (Table 3). Mothers who encountered breastfeeding difficulties during their hospital stay were more likely to receive advice to feed the baby with infant formula from hospitals (OR: 1.42; $95 \%$ CI 1.05, 1.92), modern mass media (OR: $1.54 ; 95 \%$ CI 1.21, 1.97) and family members, relatives or friends (OR: 1.60; $95 \%$ CI 1.23, 2.09) (Table 3). Moreover, mothers were more than two times as likely to receive advice of using infant formula from their family 
Public Health Nutrition

Table 2 Factors associated with receiving free samples of infant formula among mothers with infants $<6$ months old in China†, $\neq$

\begin{tabular}{|c|c|c|c|c|c|c|c|c|}
\hline & \multicolumn{2}{|c|}{ Any circumstances } & \multicolumn{2}{|c|}{ During pregnancy } & \multicolumn{2}{|c|}{ During hospital stay } & \multicolumn{2}{|c|}{ After discharge } \\
\hline & $(n 5112)$ & & $(n 5112)$ & & $(n 5112)$ & & $(n 5112)$ & \\
\hline & OR & $95 \% \mathrm{Cl}$ & OR & $95 \% \mathrm{Cl}$ & OR & $95 \% \mathrm{Cl}$ & OR & $95 \% \mathrm{Cl}$ \\
\hline \multicolumn{9}{|l|}{ Residence (reference: big cities) } \\
\hline Small and medium cities & 0.94 & $0.52,1.70$ & $1.96^{*}$ & $1 \cdot 14,3.38$ & 1.44 & $0.72,2.88$ & 0.41 & $0.15,1 \cdot 12$ \\
\hline Non-poor rural areas & 1.76 & $0.64,4.82$ & $4 \cdot 65^{\star *}$ & $1 \cdot 65,13 \cdot 14$ & $2 \cdot 06^{*}$ & $1.04,4.09$ & 0.45 & $0.17,1.19$ \\
\hline Poor rural areas & 0.56 & $0.19,1.70$ & 0.98 & $0.30,3 \cdot 19$ & 1.55 & $0.63,3.80$ & $0 \cdot 14^{\star \star *}$ & $0.05,0.41$ \\
\hline \multicolumn{9}{|l|}{ Parental characteristics } \\
\hline \multicolumn{9}{|l|}{ Maternal age (reference: $\leq 25$ years) } \\
\hline $26-35$ years & 0.97 & $0.81,1.17$ & 1.04 & $0.82,1.31$ & 0.88 & $0.65,1.18$ & 1.03 & $0.73,1.44$ \\
\hline $36+$ years & 0.72 & $0.50,1.02$ & 0.77 & $0.41,1.44$ & 0.77 & $0.45,1.33$ & 0.77 & $0.49,1.21$ \\
\hline \multicolumn{9}{|l|}{ Maternal ethnicity (reference: others) } \\
\hline Han & $1.96^{*}$ & $1.07,3.58$ & $1 \cdot 31$ & $0.67,2.56$ & $3 \cdot 18^{*}$ & $1 \cdot 22,8 \cdot 27$ & $2 \cdot 24^{\star}$ & $1.05,4.75$ \\
\hline \multicolumn{9}{|l|}{ Maternal education levels (reference: university or higher) } \\
\hline High school or college & 1.01 & $0.77,1.30$ & 0.97 & $0.72,1.31$ & 0.92 & $0.54,1.56$ & 1.05 & $0.73,1.51$ \\
\hline Junior high school & 1.13 & $0.74,1.72$ & $1 \cdot 16$ & $0.95,1.41$ & 1.26 & $0.59,2.69$ & 0.88 & $0.44,1.77$ \\
\hline Primary or below & 0.72 & $0.35,1.48$ & $0.34^{*}$ & $0.13,0.86$ & 0.95 & $0.29,3.10$ & 1.09 & $0.34,3.51$ \\
\hline \multicolumn{9}{|l|}{ Maternal occupations (reference: white-collar or professionals) } \\
\hline Industry related & 0.91 & $0.71,1.18$ & 1.04 & $0.74,1.46$ & 0.67 & $0.44,1.02$ & 0.90 & $0.60,1.36$ \\
\hline Agriculture related & 0.74 & $0.44,1.26$ & $0.43^{*}$ & $0.22,0.83$ & 0.54 & $0.19,1.51$ & $1.99^{*}$ & $1.12,3.54$ \\
\hline Unemployed & 1.01 & $0.73,1.40$ & 0.88 & $0.62,1.23$ & 0.62 & $0.33,1.14$ & $1.59^{\star \star \star}$ & $1.24,2.05$ \\
\hline \multicolumn{9}{|l|}{ Paternal education levels (reference: university or higher) } \\
\hline High school or college & 1.23 & $0.94,1.62$ & 0.97 & $0.66,1.41$ & 1.39 & $0.76,2.51$ & $1.43^{*}$ & $1.02,2.00$ \\
\hline Junior high school & 0.98 & $0.77,1.24$ & 0.85 & $0.55,1.32$ & 0.84 & $0.35,2.03$ & 1.32 & $0 \cdot 82,2 \cdot 12$ \\
\hline Primary or below & 0.92 & $0.54,1.58$ & 1.08 & $0.52,2.27$ & $0.35^{*}$ & $0.12,1.00$ & 1.38 & $0.48,3.96$ \\
\hline \multicolumn{9}{|l|}{ Paternal occupations (reference: white-collar or professionals) } \\
\hline Industry related & 0.91 & $0.68,1.22$ & 0.94 & $0.67,1.33$ & 0.74 & $0.42,1.30$ & 0.94 & $0.61,1.43$ \\
\hline Agriculture related & $0.67^{* *}$ & $0.51,0.87$ & 0.72 & $0.46,1.15$ & 1.26 & $0.79,2.01$ & $0.21^{* \star *}$ & $0.13,0.35$ \\
\hline Unemployed & 0.65 & $0.42,1.00$ & $0.58^{*}$ & $0.35,0.96$ & 0.28 & $0.06,1.24$ & 0.83 & $0.36,1.93$ \\
\hline \multicolumn{9}{|l|}{ Child characteristics } \\
\hline The first birth & 1.01 & $0.85,1.19$ & 0.91 & $0.71,1.16$ & 0.99 & $0.68,1.45$ & 1.18 & $0.88,1.59$ \\
\hline The child was male§ & 1.06 & $0.93,1.20$ & & & 1.00 & $0.71,1.42$ & $1 \cdot 16$ & $0.96,1.40$ \\
\hline Age in monthsll & $1.07^{\star \star}$ & $1.02,1.13$ & & & & & $1 \cdot 10^{\star}$ & $1.00,1.21$ \\
\hline \multicolumn{9}{|l|}{ Perinatal characteristics } \\
\hline $\begin{array}{l}\text { Had antenatal visits } \\
\text { Health facilities the mothers gave births (reference: at national } \\
\text { or provincial levels) }\end{array}$ & 0.99 & $0 \cdot 71,1 \cdot 39$ & 1.91 & $0 \cdot 87,4 \cdot 20$ & $0.44^{\star *}$ & $0.26,0.73$ & $1 \cdot 11$ & $0.55,2 \cdot 25$ \\
\hline Municipal level & 0.73 & $0.47,1.14$ & 0.95 & $0.57,1.56$ & 0.78 & $0.33,1.84$ & $0.51^{*}$ & $0.29,0.89$ \\
\hline County level & $0.49^{\star \star \star}$ & $0.34,0.72$ & 0.55 & $0.28,1.06$ & 0.65 & $0.31,1.35$ & $0.41^{\star \star \star}$ & $0.26,0.63$ \\
\hline Others (mostly private facilities) & $2 \cdot 20^{\star *}$ & $1.22,3.97$ & 1.64 & $0.64,4.22$ & $2 \cdot 72$ & $0.85,8.75$ & $1.74^{\star}$ & $1.07,2 \cdot 84$ \\
\hline Caesarean births & 1.22 & $1.00,1.49$ & & & $1 \cdot 18$ & $0.94,1.49$ & 0.99 & $0.75,1.30$ \\
\hline Long stay in health facility after births $† \dagger$ & 0.90 & $0.61,1.32$ & & & 0.92 & $0.69,1.24$ & 0.64 & $0.32,1.31$ \\
\hline 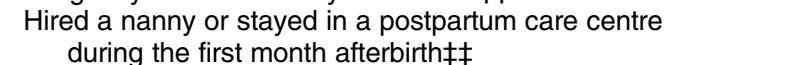 & 0.88 & $0.41,1.92$ & & & & & 0.40 & $0.09,1.72$ \\
\hline
\end{tabular}

†Values are adjusted $\mathrm{OR}$ and $95 \% \mathrm{Cl}$ from survey multivariate logistic regression models.

¥Significantly different from the null value (OR of 1 ): ${ }^{\star} P<0.05,{ }^{\star \star} P<0.01,{ }^{\star \star \star}{ }^{\star \star} P<0.001$.

$\S$ Gender of the child was not controlled for since it was unknown during pregnancy.

IIAge in months was not controlled for since it is not related to receiving free samples of infant formula during pregnancy and hospital stay.

TCaesarean births were not controlled for since it was unknown during pregnancy.

††Long stay in health facility after births: vaginal births: $\geq 4 \mathrm{~d}$; caesarean births: $\geq 7 \mathrm{~d}$. It was not controlled for since it was unknown during pregnancy.

††Hired a nanny or stayed in a postpartum care centre during the first month afterbirth was not controlled for since it happened after discharge. 
Table 3 Associate factors of receiving advice to feed the baby with infant formula among mothers with infants $<6$ months old in Chinat, $\neq$

Family members

\begin{tabular}{|c|c|c|c|c|c|c|c|c|c|c|}
\hline & \multicolumn{2}{|c|}{$\begin{array}{l}\text { Any sources/circum- } \\
\text { stances (n 5112) }\end{array}$} & \multicolumn{2}{|c|}{ Hospitals ( $n$ 5112) } & \multicolumn{2}{|c|}{$\begin{array}{l}\text { Traditional mass } \\
\text { media§ }(n 5112)\end{array}$} & \multicolumn{2}{|c|}{$\begin{array}{l}\text { Modern mass mediall } \\
(n 5112)\end{array}$} & \multicolumn{2}{|c|}{$\begin{array}{l}\text { Family members, } \\
\text { relatives or friend } \\
(n 5112)\end{array}$} \\
\hline & OR & $95 \% \mathrm{Cl}$ & OR & $95 \% \mathrm{Cl}$ & OR & $95 \% \mathrm{Cl}$ & OR & $95 \% \mathrm{Cl}$ & OR & $95 \% \mathrm{Cl}$ \\
\hline \multicolumn{11}{|l|}{ Residence (reference: big cities) } \\
\hline Small and medium cities & 0.83 & $0.45,1.53$ & 0.65 & $0.38,1.11$ & 0.57 & $0.27,1.21$ & $1.84^{\star *}$ & $1 \cdot 20,2 \cdot 80$ & 0.94 & $0.29-3.00$ \\
\hline Non-poor rural areas & 0.69 & $0.34,1.39$ & $0.37^{\star \star \star}$ & $0.21,0.66$ & $0.17^{\star \star \star}$ & $0.06,0.48$ & 0.84 & $0.44,1.62$ & 1.31 & $0.52-3.31$ \\
\hline Poor rural areas & 0.73 & $0.39,1.36$ & $0.35^{\star}$ & $0.13,0.91$ & 0.51 & $0.20,1.28$ & $1 \cdot 19$ & $0.73,1.94$ & 1.80 & $0.67-4.84$ \\
\hline \multicolumn{11}{|l|}{ Parental characteristics } \\
\hline \multicolumn{11}{|l|}{ Maternal age (reference: $\leq 25$ years) } \\
\hline $26-35$ years & 1.14 & $0.93,1.40$ & $1 \cdot 11$ & $0.80,1.54$ & 1.33 & $0.87,2.02$ & $1 \cdot 12$ & $0.80,1.56$ & $1 \cdot 10$ & $0.73-1.64$ \\
\hline $36+$ years & 1.25 & $0.93,1.67$ & $1 \cdot 18$ & $0.75,1.87$ & $2 \cdot 13^{\star \star *}$ & $1.36,3.34$ & 1.20 & $0.77,1.87$ & 0.65 & $0.34-1.26$ \\
\hline \multicolumn{11}{|l|}{ Maternal ethnicity (reference: others) } \\
\hline Han & $1 \cdot 30$ & $0.93,1.81$ & 1.51 & $0.92,2.49$ & $1 \cdot 13$ & $0.61,2.09$ & $1.61^{\star \star}$ & $1 \cdot 15,2 \cdot 27$ & 0.62 & $0.32-1.23$ \\
\hline \multicolumn{11}{|l|}{$\begin{array}{l}\text { Maternal education levels (reference: } \\
\text { university or higher) }\end{array}$} \\
\hline High school or college & 1.08 & $0.91,1.28$ & $1 \cdot 15$ & $0.80,1.66$ & 1.38 & $0.80,2.38$ & 1.02 & $0.68,1.53$ & 0.89 & $0.60-1.30$ \\
\hline Junior high school & 0.90 & $0.71,1 \cdot 15$ & $1 \cdot 11$ & $0.79,1.57$ & 1.12 & $0.51,2.43$ & 0.73 & $0.43,1.23$ & 0.70 & $0.27-1.80$ \\
\hline Primary or below & $0.65^{\star}$ & $0.44,0.96$ & 1.23 & $0.75,2.00$ & 0.72 & $0.24,2 \cdot 18$ & 0.49 & $0.23,1.07$ & 0.65 & $0.20-16$ \\
\hline \multicolumn{11}{|l|}{$\begin{array}{l}\text { Maternal occupations (reference: } \\
\text { white-collar or professionals) }\end{array}$} \\
\hline Industry related & $1 \cdot 14$ & $0.82,1.58$ & 0.90 & $0.50,1.63$ & 0.94 & $0.43,2.06$ & $1.53^{\star \star \star}$ & $1.23,1.91$ & $1.66^{*}$ & $1.02-2 \cdot 72$ \\
\hline Agriculture related & 0.74 & $0.46,1.21$ & 0.93 & $0.46,1.86$ & 0.63 & $0.23,1.71$ & 1.17 & $0.67,2.03$ & 0.50 & $0.13-1.94$ \\
\hline Unemployed & 0.99 & $0.81,1.21$ & 0.97 & $0.75,1.27$ & 0.88 & $0.66,1.17$ & $1 \cdot 19$ & $0.91,1.57$ & 1.21 & $0.77-1.90$ \\
\hline \multicolumn{11}{|l|}{$\begin{array}{l}\text { Paternal education levels (reference: } \\
\text { university or higher) }\end{array}$} \\
\hline High school or college & 1.26 & $0.90,1.77$ & 1.29 & $0.89,1.88$ & 0.90 & $0.66,1.22$ & 1.08 & $0.72,1.61$ & 1.09 & $0.46-2.57$ \\
\hline Junior high school & $1 \cdot 10$ & $0.71,1.72$ & $1 \cdot 11$ & $0.69,1.78$ & 0.84 & $0.41,1.71$ & 1.09 & $0.68,1.73$ & 0.86 & $0.25-2.89$ \\
\hline Primary or below & 1.34 & $0.75,2.39$ & 1.47 & $0.68,3.17$ & 0.55 & $0.19,1.61$ & 1.05 & $0.54,2.05$ & 0.40 & $0.06-2.91$ \\
\hline \multicolumn{11}{|l|}{$\begin{array}{l}\text { Paternal occupations (reference: } \\
\text { white-collar or professionals) }\end{array}$} \\
\hline Industry related & 0.86 & $0.72,1.02$ & 0.86 & $0.68,1.08$ & $1.44^{\star *}$ & $1 \cdot 10,1 \cdot 89$ & 0.87 & $0.64,1.19$ & 0.86 & $0.58-1.27$ \\
\hline Agriculture related & $0.59^{\star \star}$ & $0.43,0.81$ & 0.80 & $0.47,1.37$ & 0.77 & $0.28,2 \cdot 13$ & $0.46^{\star \star \star}$ & $0.31,0.71$ & 0.41 & $0.10-1.72$ \\
\hline Unemployed & 0.74 & $0.47,1.15$ & 0.78 & $0.36,1.67$ & 0.69 & $0.32,1.49$ & $0.43^{*}$ & $0.22,0.84$ & 1.65 & $0.63-4.31$ \\
\hline \multicolumn{11}{|l|}{ Child characteristics } \\
\hline The first birth & 1.20 & $0.96,1.51$ & $1 \cdot 13$ & $0.91,1.39$ & 1.30 & $0.89,1.88$ & 1.17 & $0.93,1.47$ & 1.24 & $0.71-2.15$ \\
\hline The child was male & 1.03 & $0.90,1.18$ & $1 \cdot 11$ & $0.88,1.38$ & 1.01 & $0.78,1.29$ & 0.93 & $0.79,1.08$ & 1.04 & $0.78-1.39$ \\
\hline Age in months & $1 \cdot 10^{\star *}$ & $1.03,1.18$ & 1.00 & $0.92,1.08$ & 1.09 & $0.99,1.19$ & $1 \cdot 12^{\star \star}$ & $1.04,1 \cdot 19$ & $1 \cdot 21^{\star \star \star}$ & $1 \cdot 10-1 \cdot 33$ \\
\hline \multicolumn{11}{|l|}{ Perinatal characteristics } \\
\hline Had antenatal visits & $1 \cdot 20$ & $0.86,1.68$ & 0.77 & $0.46,1 \cdot 28$ & 1.00 & $0.44,2 \cdot 26$ & 1.15 & $0.87,1.52$ & $2 \cdot 33$ & $0.75-7 \cdot 23$ \\
\hline \multicolumn{11}{|l|}{$\begin{array}{l}\text { Health facilities the mothers gave births } \\
\text { (reference: at national or provincial } \\
\text { levels) }\end{array}$} \\
\hline Municipal level & $0.76^{*}$ & $0.61,0.96$ & $0.66^{\star \star \star}$ & $0.53,0.82$ & 1.02 & $0.59,1.76$ & $0.58^{\star \star}$ & $0.38,0.86$ & $1 \cdot 77^{*}$ & $1 \cdot 10-2 \cdot 85$ \\
\hline County level & $0.64^{\star *}$ & $0.47,0.86$ & $0.58^{\star \star \star}$ & $0.43,0.78$ & 0.69 & $0.44,1.08$ & $0.37^{\star \star *}$ & $0.24,0.55$ & $2 \cdot 26^{*}$ & $1.03-4.92$ \\
\hline Others (mostly private facilities) & $1 \cdot 11$ & $0.50,2.47$ & 0.63 & $0.30,1.31$ & $0.34^{\star \star *}$ & $0.24,0.47$ & $0.32^{\star \star \star}$ & $0.17,0.60$ & 0.47 & $0.12-1.87$ \\
\hline Caesarean births & 1.21 & $0.97,1.52$ & 1.15 & $0.86,1.54$ & 1.06 & $0.84,1.35$ & 0.98 & $0.68,1.41$ & 1.22 & $0.92-1.64$ \\
\hline
\end{tabular}




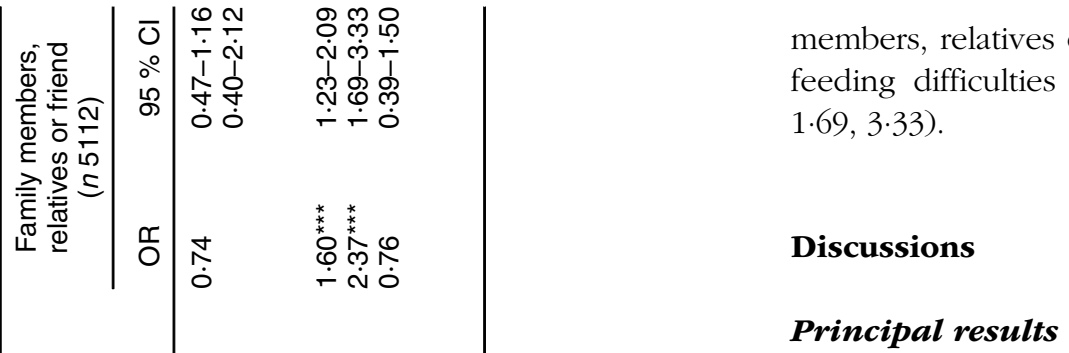

In the current study, we found that the promotion of infant formula in the form of free samples and advice was common in China. Mothers reported receiving infant formula promotion from multiple channels and at different time points including during pregnancy, hospital stay and after discharge.

First, the likelihood of receiving free samples of infant formula was still high (about 20\% in big cities and 16\% overall). This likelihood is lower than what has been found in previous studies in six big cities in China ${ }^{(20)}$. In a sample of almost 300 mothers recruited from outpatient clinics in seventeen hospitals in 2012, previous study documented that $40 \%$ of the mothers received free samples of infant formula; $76 \%$ received samples in or near hospitals, mostly by BMS sale representatives (61\%) and health workers $(37 \%)^{(20)}$. Our study was a population-based study across the country with a sample size of more than 5000 mothers, and thus the findings might not be readily comparable to the hospital-based studies in big cities. The lower prevalence of receiving free samples of infant formula, however, might indicate a certain progress towards promoting breast-feeding in China, including strengthening the baby-friendly environment through baby-friendly hospital reassessment ${ }^{(34)}$ and the implementation of early essential newborn care (EENC) ${ }^{(35,36)}$. Most of the hospitals participating in the studies ${ }^{(20)}$ had been accredited as baby-friendly hospitals and thus would have good background to shift back to a supportive environment for breast-feeding. A recent report by China Consumers Association on the promotion and sales of BMS revealed that mothers who gave birth to their babies in non-baby-friendly hospitals were more likely to use $\mathrm{BMS}^{(37)}$. In addition, introduction of EENC in the last decade in China may have helped to improve early and exclusive breast-feeding practices in the hospitals ${ }^{(35)}$, leading to a decline in the use of infant formula and support continuing breast-feeding ${ }^{(38)}$.

In addition, the infant formula promotion in terms of providing free samples in China might have expanded to target mothers in lower socio-economic groups and in rural areas. For example, our data showed that more mothers in small and medium cities and non-poor rural counties received free samples compared with mothers in big cities during pregnancy and their hospital stay. The proportion of mothers who received free samples after hospital discharge was about the same across all areas. Socio-economic status of the mothers and fathers was not a factor, suggesting that BMS manufacturers and distributors are expanding their focus to the whole population. More importantly, the 
likelihood of mothers receiving free samples of infant formula after discharge was higher in mothers working in agriculture or unemployed than those working as white-collar or professionals. Compared with mothers with university or higher education level, the likelihood of receiving free samples of infant formula after discharge was higher in mothers with high school or college education level. By introducing free samples of infant formula in various locations and time points, the BMS manufacturers and distributors might increase the use and dependence on infant formula ${ }^{(39)}$.

Second, we found that one of every four mothers received advice to feed the baby with infant formula. This prevalence was higher when they gave birth in national or provincial hospitals. In China, patients tended to pursue higher quality healthcare services, including births in tertiary and secondary hospitals ${ }^{(40)}$. To reach more mothers, BMS manufacturers and distributors would target national and provincial hospitals and their health staff, who then might promote infant formula or tolerate violations ${ }^{(7,20)}$. For example, a study in Hangzhou and Shenzhen cities revealed that around $60 \%$ of health workers recommended infant formula to mothers ${ }^{(7)}$. Vague statements about the benefits of infant formula might have been used to impose it as a 'solution' for vulnerable mothers (e.g., mental distress, pressure of feeding and caring for her child) ${ }^{(41)}$. Giving advice to feed the baby with infant formula might be an easy 'solution' for health staff who did not have enough time, knowledge and/or skills to support a mother to successfully breastfeed her infant ${ }^{(41)}$. A study in Wuhan revealed that only one of every five female physicians and nurses received any breast-feeding training and coaching after graduation and their knowledge was surprisingly poor ${ }^{(42)}$. Breast-feeding education, training and coaching programmes in both China and other countries have proven to be effective in improving knowledge and practice of health staff ${ }^{(43,44)}$. It is essential to build capacity of health staff to support mothers from pregnancy, during their hospital stay and after discharge.

Moreover, mothers who encountered breast-feeding difficulties during their hospital stay were more likely to receive advice to feed the baby with infant formula from hospitals, modern mass media as well as their family members, relatives or friends. Those mothers greatly needed support to overcome breast-feeding challenge. As discussed above, a lack of time, capacity and skills of health workers to support breast-feeding might explain the higher prevalence of receiving advice of feeding infant formula from hospitals. Advice of feeding infant formula may come from multiple sources through modern mass media. First, BMS manufacturers and distributors are using modern mass media to reach more mothers and fathers. The BMS manufacturers and distributors might still make use of existing traditional mass media in cities such as TV, radio, magazines and books. An adjusted logistic regression model showed that the prevalence of receiving advice to feed the baby with infant formula from modern mass media in big cities was as high as that in non-poor and poor rural counties but lower than that in small and medium cities. Predominant platforms of modern mass media were WeChat moments or QQ chats (50.6\%), maternal and baby product malls (49.4\%) and organisations' or companies' websites, WeChat or Weibo (37.8\%). A recent study also found many infant formula promotions on parenting apps in China ${ }^{(15)}$. Inappropriate promotion of BMS products through modern mass media is difficult to monitor and sanction compared with traditional mass media ${ }^{(45,46)}$. Specifically, mothers may get advice of infant formula feeding from social media. China has the world's largest number of internet users and most active environment for social media $^{(47)}$. Social media is a good channel in China as computers, tablets, smart phones and internet plans are accessible and affordable, as well as popular among members of younger generations ${ }^{(48)}$. Infant feeding advice from family members (especially spouses) and friends might shape the practices of the mothers directly or indirectly through daily interactions and use of social media. Thus, future interventions should include immediate social networks of mothers especially their family members ${ }^{(49,50)}$.

Third, since abolishment of the Measure in 2017, only the Maternal and Infant Health Care Law and its Implementation Measures ${ }^{(51)}$ and the Advertising Law ${ }^{(52)}$ have provisions regarding the regulation of the inappropriate marketing of BMS in China. There are still major gaps in these two laws compared with the requirements of the Code and in view of the actual needs for regulating the marketing of BMS in China. The current report from WHO, UNICEF and IBFAN shows that policies in China cover only few provisions of the Code and has big gaps in informational/educational materials, promotion to general public, promotion in health care facilities, engagement with health workers and systems, labelling, monitoring and enforcement ${ }^{(24)}$. China would benefit from strengthening policies and regulations in alignment with the Code because it would create a legal corridor for breast-feeding promotion, protection and supports in all settings ${ }^{(4)}$. For example, giving free sample and advice on using BMS in health facilities and point-of-sales would then become illegal and be regulated ${ }^{(23,24)}$. In addition to regulating traditional media, modern mass media should be regulated too ${ }^{(4,46)}$. To monitor contents in modern mass media, maximising the role of international and governmental organisations, civil society groups in report violations can be an effective measure ${ }^{(4,46)}$. Given BMS companies are using and sponsoring groups or individuals to use modern media channels to promote their products, media firms should ensure the contents are compliant to the Code and national regulations ${ }^{(46,53)}$.

\section{Strengths and limitations}

Compared with previous studies, the current study has several strengths. Firstly, by using data from a large sample of 
mothers from various locations and of different socioeconomic status, it provides updated information on the marketing of BMS in China and compares promotional tactics of BMS manufacturers and distributors across different locations. Secondly, limiting the sample to mothers of infants 0-5.9 months reduced recall bias of exposure to infant formula promotion. However, the current study also faced several limitations. Health systems in twelve sample sites selected in the first stage have comparatively higher levels of executive capacity, which may be correlated with their capacity to provide a better supporting environment for maternal and child health. Thus, cautions are needed when generalising results suggested in the current study nationally and comparing these results with existing literature. Second, responses of mothers may still suffer from recall bias and social desirability bias. Third, the cross-sectional nature of the current study might suggest the possible associations between the place of residence or socio-economic status with the exposure to infant formula promotion rather than confirm the association. However, the reverse association would be small: it is more likely that a BMS manufacturer or distributor targets a certain group of mothers than exposure to infant formula promotion changes the place of residence or socio-economic status of the mothers. Fourth, although we capture key information relating to BMS, the content of our questionnaire was not as exhaustive as the complete list of the WHO's NetCode Assessment Module ${ }^{(54)}$. Nonetheless, we captured two main aspects of infant formula promotion: free samples and advice to feed the baby with infant formula. Also, several questions are left unanswered by the survey. For example, it is not clear who recommended the formula in the hospitals, whether they received antenatal and postnatal care at the same hospital where they deliver.

\section{Conclusions}

In conclusion, the promotion of infant formula in the form of free samples and advice is common in surveyed areas in China. The promotion has targeted women from different socio-economic groups, place of residence and at various time points such as during pregnancy, delivery, postnatal period and beyond. The study findings would suggest the need to strengthen regulation and enforcement of the Code that restricts the promotion of BMS, especially within health facilities and through modern forms of media. The local government, health sector, media, civil groups, mothers and family members in less urbanised areas should also be prepared to protect recommended breast-feeding practices and against the negative effect of BMS promotion.

\section{Acknowledgements}

Acknowledgements: We are grateful to Joy Del Rosso, Jessica Escobar-Alegria, Sujata Bose and Andres Martinez from Alive \& Thrive/FHI 360 Headquarters for their comments and suggestions to improve this manuscript. Financial support: The study was partially funded by the Bill \& Melinda Gates Foundation to China Development Research Foundation (grant number OPP1152715) and Alive \& Thrive (grant number OPP50838). Conflict of interest: None of the authors had a conflict of interest related to any part of the current study or manuscript. Authorship: The authors' responsibilities were as follows: J.L., T.T.N. and Z.Y. designed the study; Y.D. and Z.Y. collected the data; J.L. and T.T.N. analysed the data; J.L., T.T.N., Z.Y. and R.M. interpreted the data; J.L. and T.T.N. drafted this manuscript and R.M., Y.D. and Z.Y. provided critical intellectual feedback to help revise the manuscript. All authors have read and approved the final manuscript. Ethics of buman subject participation: The current study was conducted according to the guidelines laid down in the Declaration of Helsinki and all procedures involving research study participants were approved by the Medical Research Ethnics Committee at the National Institute for Nutrition and Health at the Chinese Center for Disease Control and Prevention (NINH, China CDC). Written informed consent was obtained from all subjects.

\section{Supplementary material}

For supplementary material accompanying this paper please visit https://doi.org/10.1017/S1368980020005364

\section{References}

1. Piwoz EG \& Huffman SL (2015) The impact of marketing of breast-milk substitutes on who-recommended breastfeeding practices. Food Nutr Bull 36, 373-386.

2. WHO \& UNICEF (2019) Cross-Promotion of Infant Formula and Toddler Milks Information Note. Geneva: World Health Organization.

3. Rollins NC, Bhandari N, Hajeebhoy N et al. (2016) Why invest, and what it will take to improve breastfeeding practices? Lancet 387, 491-504.

4. Robinson H, Buccini G, Curry L et al. (2019) The World Health Organization Code and exclusive breastfeeding in China, India, and Vietnam. Matern Child Nutr 15, e12685.

5. McFadden A, Mason F, Baker J et al. (2016) Spotlight on infant formula: coordinated global action needed. Lancet 387, 413-415.

6. Nguyen TT, Withers M, Hajeebhoy $\mathrm{N}$ et al. (2016) Infant formula feeding at birth is common and inversely associated with subsequent breastfeeding behavior in Vietnam. $J$ Nutr 146, 2102-2108.

7. Tang L, Lee AH, Binns CW et al. (2014) Widespread usage of infant formula in China: a major public health problem. Birth 41, 339-343.

8. Li J, Nguyen TT, Wang Xet al. (2020) Breastfeeding practices and associated factors at the individual, family, health facility and environmental levels in China. Matern Child Nutr 16, e13002.

9. Victora CG, Bahl R, Barros AJD et al. (2016) Breastfeeding in the 21st century: epidemiology, mechanisms, and lifelong effect. Lancet 387, 475-490. 
10. Baker P, Smith J, Salmon L et al. (2016) Global trends and patterns of commercial milk-based formula sales: Is an unprecedented infant and young child feeding transition underway? Public Health Nutr 19, 2540-2550.

11. Kent G (2015) Global infant formula: monitoring and regulating the impacts to protect human health. Int Breastfeed J 10, 1-2.

12. Euromonitor International (2015) Baby Food in China. https://www.euromonitor.com/baby-food-in-china/report (accessed June 2019).

13. Euromonitor International (2018) Baby Food in China. https://www.euromonitor.com/baby-food-in-china/report (accessed June 2019).

14. Rothstein JD, Caulfield LE, Broaddus-Shea ET et al. (2020) "The doctor said formula would help me": health sector influences on use of infant formula in peri-urban Lima, Peru. Soc Sci Med 244, 112324.

15. Zhao J, Li M \& Freeman B (2019) A baby formula designed for Chinese Babies: content analysis of milk formula advertisements on Chinese parenting apps. JMIR mHealth uHealth 7, e14219.

16. Appleton J, Laws R, Russell CG et al. (2018) Infant formula feeding practices and the role of advice and support: an exploratory qualitative study. BMC Pediatr 18, 12 .

17. Appleton J, Fowler C, Laws R et al. (2020) Professional and non-professional sources of formula feeding advice for parents in the first six months. Matern Child Nutr 13, e12942.

18. Howard C, Howard F, Lawrence R et al. (2000) Office prenatal formula advertising and its effect on breast-feeding patterns. Obstet Gynecol 95, 296-303.

19. Howard CR, Howard FM \& Weitzman ML (1994) Infant formula distribution and advertising in pregnancy: a hospital survey. Birth 21, 14-19.

20. Liu A, Dai Y, Xie X et al. (2014) Implementation of international code of marketing breast-milk substitutes in China. Breastfeed Med 9, 467-472.

21. Tang L, Binns CW \& Lee AH (2015) Infant formula crisis in China: a cohort study in Sichuan province. J Health Popul Nutr 33, 117-122.

22. Brady JP (2012) Marketing breast milk substitutes: problems and perils throughout the world. Arch Dis Child 97, 529-532.

23. World Health Organization (1981) International Code of Marketing of Breastmilk Substitutes. Geneva: WHO.

24. WHO, UNICEF \& IBFAN (2020) Marketing of breast milk substitutes: national implementation of the international code, status report 2020. https://www.who.int/publications/i/ item/9789240006010 (accessed July 2020).

25. Ministry of Health (1995) Administrative Measures for the Marketing of Breastmilk Substitutes. http://www.nhc.gov. cn/fys/s7899/200804/0c5e174e5f9a461e825c4c556a735ace. shtml (accessed July 2020).

26. China Food and Drug Administration (2016) Administrative Measures for the Registration of Recipes for Formula Powder Products for Infants and Young Children (CFDA Decree 26). http://samr.cfda.gov.cn/WS01/CL1197/155260. html (accessed May 2019).

27. Bloomberg (2018) China Removes 1,400 Baby Formula Products From Shelves. https://www.bloomberg.com/ news/articles/2018-01-04/china-s-baby-formula-revamp-maytrigger-boom-for-nestle-danone (accessed July 2020).

28. Chen M \& Hu Y (2017) Infant formula maker Mead to feed rural areas. China Dly. http://www.chinadaily.com.cn/ newsrepublic/2017-10/30/content_33909850.htm (accessed May 2019).

29. Zeng Y \& Hesketh T (2016) The effects of China's universal two-child policy. Lancet 388, 1930-1938.

30. State Council of the People's Republic of China (2014) Administrative Division. http://english.www.gov.cn/ archive/china_abc/2014/08/27/content_281474983873401.htm (accessed August 2014).
31. Ministry of Civil Affairs of the People's Republic of China (2013) Statistical Table of Administrative Divisions of the People's Republic of China. http://xzqh.mca.gov.cn/ statistics/ (accessed May 2020).

32. The State Council Leading Group Office of Poverty Alleviation and Development (2014) List of national poor counties. http://www.cpad.gov.cn/art/2014/12/23/art_343_ 981.html (accessed December 2014).

33. Campbell OMR, Cegolon L, Macleod D et al. (2016) Length of stay after childbirth in 92 countries and associated factors in 30 low- and middle-income countries: compilation of reported data and a cross-sectional analysis from nationally representative surveys. PLoS Med 13, e1001972.

34. National Health and Family Planning Commission of the People's Republic of China (2014) Notice on the Reassessment of Baby-Friendly Hospitals. http://www.nhc. gov.cn/xxgk/pages/viewdocument.jsp?dispatchDate $=\&$ staticUrl=/fys/s3585/201406/556c0b7673e8470f9641c 28d119a9f31.shtml\&wenhao=\&x56FD;\&x536B;\&x5987;\&x5E 7C;\&x51FD;(2014)185\&x53F7;\&utitle=\&x56FD;\&x5BB6;\&x536 B;\%E7 (accessed December 2019).

35. Xu T, Yue Q, Wang Y et al. (2018) Childbirth and early newborn care practices in 4 provinces in China: a comparison with WHO recommendations. Glob Heal Sci Pract 6, 565-573.

36. Li Z, Mannava P, Murray JCS et al. (2020) Association between early essential newborn care and breastfeeding outcomes in eight countries in Asia and the Pacific: a cross-sectional observational -study. BMJ Glob Heal 5, e002581.

37. China Consumers Association (2019) 2018 report on the promotion and sales of breast-milk substitute. http://www.cqn. com.cn/pp/content/2019-03/07/content_6859399.htm (accessed May 2020) (in Chinese).

38. Qiu L, Zhao Y, Binns CW et al. (2009) Initiation of breastfeeding and prevalence of exclusive breastfeeding at hospital discharge in urban, suburban and rural areas of Zhejiang China. Int Breastfeed J 4, 1.

39. Rosenberg KD, Eastham CA, Kasehagen LJ et al. (2008) Marketing infant formula through hospitals: the impact of commercial hospital discharge packs on breastfeeding. Am J Public Health 98, 290-295.

40. Wang H, Zhang D, Hou Z et al. (2018) Association between social health insurance and choice of hospitals among internal migrants in China: a national cross-sectional study. $B M J$ Open 8, e018440.

41. Munblit D, Crawley H, Hyde R et al. (2020) Health and nutrition claims for infant formula are poorly substantiated and potentially harmful. BMJ 369, m875.

42. Ouyang Y, Xu Y \& Zhang Q (2012) Survey on breastfeeding among Chinese female physicians and nurses. Nurs Health Sci 14, 298-303.

43. Wallace LM, Ma Y, Qiu LQ et al. (2018) Educational videos for practitioners attending Baby Friendly Hospital Initiative workshops supporting breastfeeding positioning, attachment and hand expression skills: effects on knowledge and confidence. Nurse Educ Pract 31, 7-13.

44. Valdes V, Pugin E, Nurse-Midwife R et al. (1995) The effects on professional practices of a three-day course on breastfeeding. J Hum Lact 11, 185-190.

45. Eagle L \& Dahl S (2018) Product placement in old and new media: examining the evidence for concern.J Bus Ethics 147, 605-618.

46. Vinje KH, Phan LTH, Nguyen TT et al. (2017) Media audit reveals inappropriate promotion of products under the scope of the International Code of Marketing of Breast-Milk Substitutes in South-East Asia. Public Health Nutr 20, 1333-1342.

47. Chiu C, Ip C \& Silverman A (2012) Understanding social media in China. McKinsey $Q \mathbf{2}, 78-81$.

48. UNICEF (2017) State of the Worlds Children 2017-Children in a Digital World. New York: Unicef. 
49. Bai DL, Fong DYT, Lok KYW et al. (2016) Relationship between the infant feeding preferences of Chinese mothers' immediate social network and early breastfeeding cessation. J Hum Lact [Internet] 32, 301-308.

50. Abbass-Dick J, Brown HK, Jackson KT et al. (2019) Perinatal breastfeeding interventions including fathers/partners: a systematic review of the literature. Midwifery 75, 41-51.

51. State Council of the People's Republic of China (2001) Implementation Measures of the Maternal and Infant Health Care Law. http://www.gov.cn/banshi/2005-08/01/ content_19126.htm (accessed May 2020).
52. National People's Congress of China (2015) Advertising Law of the People's Republic of China. http://www.gov.cn/ zhengce/2015-04/25/content_2853642.htm (accessed May 2020).

53. Collin J, Ralston R, Hill S et al. (2020) Signalling Virtue, Promoting Harm: Unhealthy commodity industries and COVID-19. https://ncdalliance.org/resources/signallingvirtue-promoting-harm (accessed October 2020).

54. WHO \& UNICEF (2017) NetCode Toolkit. Monitoring the Marketing of Breast-Milk Substitutes: Protocol for Periodic Assessment. Geneva: World Health Organization. 